\title{
Estimulação imunológica em portadores do Vírus da Imunodeficiência Humana (HIV) pela prática de exercício físico
}

\author{
Immunological stimulation in Human Immunodeficiency Virus (HIV) carriers through \\ physical exercise
}

\section{Estimulación inmunológica en portadores del Virus de la Inmunodeficiencia Humana (HIV) a través del ejercicio físico}

Diovana Edna Barbosa Gomes ${ }^{1 *}$, Beatriz Sales Querente ${ }^{1}$, Maria Paula Santana Marques ${ }^{1}$, Luiza Prado Jorge $^{1}$, Ana Beatriz Rodrigues dos Santos ${ }^{1}$, Thamilis Costa Andrade ${ }^{1}$, Lara Santiago ${ }^{2}$, Bárbara Rocha Aguilar $^{3}$, Manuelly Maísa Antunes Guimarães Pereira ${ }^{4}$, Geraldo Lino da Silva Junior ${ }^{1}$.

\section{RESUMO}

Objetivo: Avaliar os efeitos da prática de exercício físico de diferentes tipos e intensidades sobre a estimulação do sistema imunológico nos portadores do HIV, bem como os benefícios para a saúde e bemestar dos indivíduos imunossuprimidos. Revisão bibliográfica: O HIV possui uma alta afinidade pelas células do sistema imunológico, em particular os linfócitos TCD4+, proporcionando uma diminuição na quantidade e qualidade desse complexo. Assim, além dos tratamentos medicamentosos, há também recomendações da prática de exercício físico de forma regular, que auxiliam na estimulação do sistema imunológico, bem como contribuem no controle dos efeitos adversos acarretados pela terapia antirretroviral, sem gerar sequelas no sistema imune, melhorando as medidas antropométricas e funcionais do paciente. Com isso, a atividade física deve ser indicada a afim de favorecer tanto à reconstituição do sistema imunológico, quanto uma melhora na saúde mental do indivíduo. Considerações finais: Este constructo é de fundamental importância, pois irá prover embasamento teórico-metodológico para mais pesquisas sobre a relação da atividade física e a melhora da qualidade de vida dos pacientes portadores do HIV.

Palavras-chave: HIV, AIDS, Sistema imunológico, Atividade física.

\begin{abstract}
Objective: To evaluate the effects of physical exercise practice of different types and intensities on the stimulation of the immune system in HIV carriers, as well as the benefits for the health and well-being of immunosuppressed individuals. Bibliographic review: HIV has a high affinity for the cells of the immune system, in particular the TCD4+ lymphocytes, providing a decrease in the quantity and quality of this complex. Thus, in addition to drug treatments, there are also recommendations for regular physical exercise, which help to stimulate the immune system, as well as contribute to the control of adverse effects caused by antiretroviral therapy, without generating sequelae in the immune system, improving measures anthropometric and functional characteristics of the patient. Thus, physical activity should be indicated in order to favor both the reconstitution of the immune system and an improvement in the individual's mental health. Final considerations: This construct is of fundamental importance, as it will provide a theoretical and methodological basis for further research on the relationship between physical activity and the improvement of the quality of life of patients with HIV.
\end{abstract}

Keywords: HIV, AIDS, Immune system, Physical activity.

\section{RESUMEN}

Objetivo: Evaluar los efectos de la práctica de ejercicio físico de diferentes tipos e intensidades sobre la estimulación del sistema inmunológico en portadores del VIH, así como los beneficios para la salud y el bienestar de las personas inmunodeprimidas. Revisión bibliográfica: EI VIH tiene una alta afinidad por las

\footnotetext{
${ }^{1}$ Faculdade Santo Agostinho (FASA), Vitória da Conquista - BA. *E-mail: diovana3223@gemail.com

2 Faculdade Santo Agostinho (FASA), Itabuna - BA

3 Faculdades Unidades do Norte de Minas (FUNORTE), Montes Claros - MG.

4 Faculdades Integradas Pitágoras (FIPGuanambi), Guanambi - BA.
} 
células del sistema inmunológico, en particular los linfocitos TCD4 +, lo que provoca una disminución en la cantidad y calidad de este complejo. Así, además de los tratamientos farmacológicos, también existen recomendaciones de ejercicio físico regular, que ayudan a estimular el sistema inmunológico, además de contribuir al control de los efectos adversos provocados por la terapia antirretroviral, sin generar secuelas en el sistema inmunológico, mejorando las medidas. características antropométricas y funcionales del paciente. Por tanto, la actividad física debe estar indicada para favorecer tanto la reconstitución del sistema inmunológico como una mejora de la salud mental del individuo. Consideraciones finales: Este constructo es de fundamental importancia, ya que proporcionará una base teórica y metodológica para futuras investigaciones sobre la relación entre la actividad física y la mejora de la calidad de vida de los pacientes con VIH.

Palabras clave: VIH, SIDA, Sistema inmunológico, Actividad física.

\section{INTRODUÇÃO}

O Vírus da Imunodeficiência Humana (HIV), infecta o indivíduo e ataca o sistema imunológico, provocando imunossupressão nas pessoas portadores, devido ao seu tropismo pelas células imunológicas, principalmente os linfócitos TCD4+. Esse vírus é o responsável pelo desenvolvimento da Síndrome da Imunodeficiência Adquirida (AIDS), que atualmente é mais prevalente na população jovem feminina, nas pessoas que vivem na pobreza e com baixo nível de educação formal (SILVA RAR, et al., 2016).

Atualmente cerca de 37 milhões de pessoas vivem com HIV em todo o mundo. Além disso, aproximadamente 1 milhão de pessoas morrem, por ano, sem saber que estão contaminados pelo vírus ou por começarem o tratamento tardiamente. Outrossim, nos últimos cinco anos o Brasil tem apresentado, anualmente, cerca de 39 mil casos, no entanto, esse número vem sofrendo um decréscimo desde de 2013 (DE MELO BO, et al., 2018).

A AIDS é considerada uma Infecção Sexualmente Transmissível (IST's), apesar de também possuir outras vias de transmissão, como a vertical (mãe para filho), parenteral (uso de drogas injetáveis) e o contato sanguíneo com matérias ou secreções contaminadas. Na atualidade, o tipo de prevenção considerada mais eficaz continua sendo a proteção durante o ato sexual, com o uso de preservativos (SILVA RAR, et al., 2016).

Com a aquisição da Terapia Antirretroviral (TARV), na atualidade, houve o aumento na expectativa de vida dos portadores de HIV, oferecendo a eles um novo e melhor prognóstico. Com os avanços sobre o conhecimento do ciclo de infecção e replicação do HIV, ocorreu também uma evolução no tratamento com o uso de antirretrovirais (CARVALHO PP, et al., 2019).

No Brasil, o tratamento medicamentoso é de acesso universal e de forma gratuita a toda população (FREITAS JP, et al., 2018). A terapia medicamentosa teve início a partir do seu uso como monoterapia com Zidovudina (AZT), nos anos de 1994 a 1996 que passou, posteriormente, a ser utilizada como terapia dupla, com isso passou a ser o plano terapêutico, no ano de 1996, começou a ser introduzida a terapia tríplice, que são os inibidores das proteases. Atualmente a terapêutica voltada para o combate do vírus HIV é tão eficaz que faz com que o mesmo fique quase que infectável no corpo humano, assim reduzindo a morbidade e mortalidade dos indivíduos portadores do HIV (CARVALHO PP, et al., 2019).

Ademais, o tratamento não medicamentoso, como a prática diária de exercício físico tem sido um importante fator, que proporciona aumento na qualidade e expectativa de vida dos pacientes. Além do acometimento físico, os pacientes encontram-se afetados psicologicamente, o que pode acelerar diretamente o avanço da infecção. Dessa forma, conclui-se que a atividade física pode contribuir para a manutenção adequada do estado imunológico e psicossocial do paciente. Com isso, a atividade física feita de maneira regular favorece tanto a reconstituição do sistema imunológico, quanto uma melhora na saúde psíquica (GOUVÊA-E-SILVA LF, et al., 2016).

É importante dizer que o Sistema Único de Saúde (SUS) disponibiliza para a sociedade o Programa Academia da Saúde (PAS), sendo essa uma estratégia de promoção à saúde, a qual fortalece a relação entre a Estratégia de Saúde da Família (ESF), Núcleos Ampliados de Saúde da Família (NASF) e a Vigilância em Saúde. Assim, a união desses órgãos permite a acessibilidade e a influência para a criação de programas 
educativos que favorecem a expansibilidade das práticas de exercício físico que auxiliam no condicionamento do sistema imunológico dos pacientes portadores de HIV e também ao estado psicológico dos mesmos (LAZZAROTTO AR e BAZZO KO, 2016).

Esse constructo teve como objetivo, avaliar os efeitos da prática de exercício físico de diferentes tipos e intensidades sobre a estimulação do sistema imunológico nos portadores do HIV, bem como os benefícios para a saúde e bem-estar dos indivíduos imunossuprimidos.

\section{REVISÃO BIBLIOGRÁFICA}

Atualmente a AIDS ainda é considerada uma pandemia, constituindo um dos mais sérios problemas mundiais de saúde pública. A doença em questão é causada pela infecção do HIV, que possui uma alta afinidade pelas células do sistema imunológico, em particular os linfócitos TCD4+, proporcionando uma depressão na quantidade e qualidade desse complexo (MACIEL KL, et al., 2018). Essa doença é desenvolvida cronicamente, tornando lenta e gradual a destruição do sistema imunológico, que consequentemente favorece o aparecimento de doenças oportunistas (LIMA ACMACC, et al., 2017).

A infecção pelo HIV pode ser causada por uma ou mais formas, sendo essas diferenciadas a partir da avaliação genética, mas que possuem relações antigênicas entre si. Os dois tipos conhecidos são o HIV-1 (tipo1) e o HIV-2 (tipo 2), a diferença entre eles são encontradas nos parâmetros de dispersão, patogenicidade, transmissibilidade, evolução da doença e suscetibilidade a drogas (CAMPOS KR, et al., 2015).

$\mathrm{Na}$ atualidade, no mundo há prevalência da infecção pelo tipo HIV-1, sendo esse responsável pela pandemia instalada. Contudo, o HIV-2, que possui uma menor transmissibilidade e virulência, é considerado epidêmico e endêmico na África Ocidental. Acredita-se que o HIV-1 tenha surgido na África Central, uma vez que o vírus que infectava estritamente os chimpanzés entrou em contato com o homem, dando início a disseminação global nas décadas de 70/80 (DIAS J, et al., 2020, PINTO CS, et al., 2018).

O HIV possui uma grande variabilidade, promovendo uma diversidade de classificações, isso acontece graças à divergência de nucleotídeos virais. Dessa forma, foi possível realizar a classificação em subtipos, subsubtipos e as formas recombinantes circulantes. O HIV-1 possui os grupos $M, N, O$ e $P$, sendo o grupo $M$ o mais prevalente nas infecções pelo vírus. O grupo $M$, por sua vez, possui os subtipos A, B, C, D, F. G. H. J e K. Alguns subtipos do grupo $M$ são divididos em subsubtipos, esses são o $A$ e o $F$, sendo assim, formam os subsubtipos A1 e A2, F1 e F2. Além disso, em alguns indivíduos, diferentes cepas do vírus podem unir-se de formas aleatórias e desenvolver novos perfis da doença e do vírus, chamadas de formas recombinantes circulantes (CRF) (CAMPOS KR, et al., 2015).

A transmissão sexual é a principal via de infecção em todo o mundo, sendo que nos Estados Unidos a maioria dos casos ocorre pelas relações homossexuais (principalmente entre homens), de forma geral, no mundo essa transmissão se dá a partir de relações sexuais entre casais heterossexuais. É importante destacar que outras IST's contribuem para contração do HIV, pois essas doenças são capazes de lesar a pele e mucosa dos indivíduos, que por sua vez facilitam a penetração do vírus e a sua entrada nas células humanas (LIMA ACMACC, et al., 2017).

Além disso, as mulheres têm uma maior probabilidade de serem infectadas, uma vez que elas recebem fluidos que são expelidos pelo homem (ejaculação), enquanto isso, os homens possuem essa probabilidade reduzida, pois não são os receptores quando se trata de uma relação sexual. Diante disso, para que essa ocorrência aumente e se iguale a feminina é necessário que homem possua algum veículo de entrada para o micro-organismo, como abrasão, corte ou ferimento (LIMA ACMACC, et al., 2017).

A via parenteral também funciona como um veículo de transmissão e tem grande importância em três grupos específicos: usuários de drogas injetáveis, hemofílicos tratados com fator 8 e 9 e pessoas que receberam transfusão de sangue. De todos esses grupos, o primeiro merece destaque para a sua grande prevalência, uma vez que nos outros casos, há uma queda da contaminação por essa via, já que tanto os concentrados de sangue quanto o plasma recebem tratamentos e passam por monitoração antes de serem passados a outras pessoas (DA ROSA MC, et al., 2016). 
Vale lembrar que há também a transmissão vertical, de mães para filhos que é a principal causa de AIDS em crianças. Nesse processo, há três veículos: o útero (transplacentáia); intraparto (durante o parto) e lactentes (ingestão do leite materno contaminado). Ademais, o ciclo de vida viral só é possível quando esse penetra a célula humana, pois isoladamente não é capaz de se reproduzir. As primeiras células a entrarem em contato com o vírus são aquelas que fazem parte do sistema imunológico, como os monócitos, principalmente as células dendríticas, eles atacam células que possuem o marcador CD4, preferencialmente os linfócitos, entretanto, macrófagos teciduais e células da micróglia do sistema nervoso central também são considerados alvos (LIMA ACMACC, et al., 2017).

Desde 1996, ano em que a terapia antirretroviral altamente ativa (HAART) foi introduzida, o número de pacientes que morreram de AIDS e infecções oportunistas diminuiu em dois terços (NUNES AA, et al., 2015). A aceitação e aquisição desse tratamento gerou uma alta melhora clínica dos pacientes, no entanto, os medicamentos utilizados levavam os indivíduos portadores a desenvolverem alterações cardiovasculares e endócrinas (PINTO LFS, et al., 2021).

Os medicamentos antirretrovirais são recomendados para todas as pessoas infectadas com o HIV, visto que, sem o uso dos mesmos há uma maior probabilidade de desenvolver complicações graves e irreversíveis. A pessoa deve ser instruída quanto aos riscos e benefícios oferecidos pelos medicamentos, além disso, devese informa-la que a partir do início da terapia essa não pode ser interrompida e quanto mais precoce se iniciar o tratamento, maior será a chance de obter bons resultados (NUNES JÚNIOR SS e CIOSAK SI, 2018).

A terapia medicamentosa não consegue eliminar o vírus do organismo, entretanto a carga viral diminui tanto que muitas vezes podem não ser detectadas no sangue ou em outros líquidos corporais. $O$ objetivo da TARV é reduzir a carga do HIV em um nível indetectável e restaurar a contagem de linfócitos CD4 ao normal (ERRANTE PR, et al., 2018).

O tratamento medicamentoso da AIDS é baseado no ciclo de vida do vírus, posto que é considerada uma terapia antirretroviral, ou seja, tenta reverter o mecanismo de ação dos vírus no interior das células humanas. Essa terapia é fundamental para que haja uma melhora na qualidade de vida do paciente, pois diminuem a carga viral e consequentemente a imunossupressão, além de promover, significativamente, a redução da transmissão (SOUZA HC, et al., 2019). As diferentes classes dos medicamentes são baseadas em distintos pontos do ciclo viral, a fim de impedir que este continue a sua reprodução. Dessa forma, a recomendação é que a tratamento seja feito com o uso combinado de diferentes classes medicamentosas, no intuito de aumentar a capacidade protetiva (COUTINHO MFC, et al., 2018).

Além dos tratamentos medicamentosos, há também recomendações da prática de exercício físico de forma regular, que ajudam garantir a qualidade de vida dos portadores do HIV, auxiliam na estimulação do sistema imunológico dos mesmos, bem como contribuem no controle dos efeitos adversos acarretados pelas HAART, sem gerar sequelas no sistema imune, melhorando as medidas antropométricas e funcionais do paciente (NUNES AA, et al., 2015). Os exercícios físicos devem fazer parte da rotina de um paciente infectado pelo HIV, pois eles podem ajudar a prevenir e reduzir a intensidade dos danos colaterais provocados tanto pela AIDS, quanto pelas doenças oportunistas que se desenvolvem ao longo da comorbidade (RODRIGUES DA e TOIGO AM, 2015).

Apesar da introdução da TARV ser um grande avanço para o tratamento das pessoas que convivem com o HIV o seu uso traz consigo alguns efeitos adversos que podem ser contornados a partir da prática regular de atividade física (PASSOS SMK e SOUSA LDM, 2015). Os principais pontos relacionados ao uso da TARV são o surgimento da Síndrome Lipodistrofica, a qual provoca alterações na distribuição de gordura corporal (sendo mais localizada no tronco), disfunções metabólicas, que podem resultar, posteriormente, no desenvolvimento de doenças cardiovasculares, alterações hematológicas, resistência à insulina e dislipidemia. Dessa forma, observa-se que o exercício físico feito de forma adequada pode impedir o surgimento desses efeitos colaterais (ROMANCINI JLH, et al., 2012).

É importante salientar que grande maioria dos pacientes portadores de HIV são sedentários, sendo assim há uma alta relevância quando se trata de perda de força de preensão e massa muscular nesses indivíduos. 
Outras alterações evidenciadas foram na taxa metabólica basal, que favorecem ainda mais para os agravantes proporcionados pela utilização da TARV e o aumento de forma significativa da morbidade e mortalidade ao longo do desenvolvimento da patologia. O exercício físico, realizado de forma regular, consegue amenizar as chances da evolução do paciente para quadros de lipodistrofia, bem como, a hipertensão arterial, as quais podem estar relacionadas com o tempo de infecção e de terapia antirretroviral (ROMANCINI JLH, et al., 2012).

Destaca-se que, os indivíduos sedentários possuem uma menor quantidade de massa magra comparado aos pacientes que permanecem ativos, enquanto que, os que possuem lipodistrofia guardam consigo um maior percentual de massa gorda e um decréscimo progressivo de massa magra. Pacientes ativos apresentam também um aumento na taxa do metabolismo basal e na força de preensão de forma significativa. Há evidencias de que a partir da prática diária de atividades físicas o indivíduo consiga alcançar mudanças nas suas medidas antropométricas, como por exemplo redução da cintura abdominal e da massa corporal total. Outro fator que também sobre modificação é a glicemia de jejum que sofrerá uma redução (BRITO CJ, et al., 2013)

Além disso, a perda de massa muscular também pode ser um dos problemas nos soropositivos, pois acarretam alterações morfológicas e fisiológicas, que estão relacionadas com a autoestima e autoconfiança. O exercício aeróbico tem mostrado os seus benefícios em relação ao controle do estresse e como uma técnica de relaxamento capaz de diminuir a ansiedade e os sintomas depressivos (DA COSTA KM, et al., 2018). Dessa forma, observa-se que o exercício físico feito de forma adequada pode estar diretamente relacionado com o aumento da autoestima e melhora dos sintomas psicológicos (RODRIGUES DA e TOIGO AM, 2015).

Sendo assim, as alterações na fisiologia corporal é uma consequência do uso da TARV de forma prolongada pelos infectados por HIV. É importante frisar que em conjunto com o exercício físico devem ser implementadas mudanças nos hábitos alimentares destes pacientes, para um melhor controle relacionado ao anabolismo e catabolismo de alguns nutrientes, sendo esses eficazes e fundamentais para um funcionamento ótimo do sistema imunológico, ou seja, possibilita o aumento na contagem de linfócitos TCD4+. Ademais, esse conjunto de ações podem proporcionar uma queda na hipertrigliceridemia e hipercolesteromia, que também são provocados pelo uso crônico da TARV (LIMA LRA, et al., 2017, MEDEIROS RCSC, et al., 2017).

A atividade física tem um importante papel quando se trata da defesa corporal, uma vez que ela demanda um maior aporte de oxigênio, provocando fortalecimento dos músculos respiratórios, melhorando a função e capacidade pulmonar, consequentemente, dificulta a estabilização e multiplicação de microrganismos no local. Além disso, o exercício possui uma relação diretamente proporcional à circulação sanguínea, visto que os tecidos irão necessitar de uma troca rápida gasosa mais. Com isso, a circulação acelerada promove consigo uma distribuição mais veloz e eficaz das células imunológicas, favorecendo o ataque e destruição de invasores teciduais (JUNIOR JO, et al., 2017).

Exercícios físicos geram Estresse Oxidativo (EO) e desequilíbrio homeostático entre as substancias próoxidantes e antioxidantes em qualquer indivíduo que o pratique, mas essa alteração se torna ainda maior e perceptível em um portador de HIV, pela falta dos linfócitos TCD4+, pois há redução da qualidade de ação dessas células como agente antioxidante. Os radicais livres formados no corpo humano durante a atividade física se decorrem do EO, lesando uma maior quantidade de células e tecidos, que consequentemente reduz a ativação e ação das enzimas. Dessa forma, a tentativa de controle de todo esse processo é baseada nas terapias medicamentosas conjugada com a atividade física diária e regular, para que o indivíduo portador possua uma melhor qualidade de vida (MELO BP, et al., 2017).

O aumento da transpiração também é uma consequência do exercício, esse fator está relacionado com o transporte externo mais abrupto dos resíduos metabólicos, deixando de oferecer matérias suficientes para a sobrevivência e reprodução do vírus nas células humanas. Além disso, o fortalecimento muscular é um dos principais benefícios proporcionados pelo exercício, sendo o musculo cardíaco um dos mais favorecidos, facilitando o retorno de sangue ao coração e uma melhor circulação da linfa, removendo dos tecidos impurezas e corpos estranhos, promovendo a redução na chance tanto de uma maior disseminação do vírus quanto na probabilidade de desenvolver doenças oportunistas (JUNIOR JO, et al., 2017). 
A indicação do tratamento não medicamentoso, ou seja, a prática da atividade física deve ser de intensidade leve a moderada, porque estas são as consideradas benéficas para o indivíduo portador de HIV, os exercícios de alta intensidade podem acarretar um declínio no metabolismo fisiológico do sistema imune. Os exercícios físicos portam consigo uma relação direta com a estimulação do sistema imunológico, dado que eles podem induzir a medula óssea e os linfonodos a produzirem uma maior quantidade de leucócitos ou glóbulos brancos e ainda podem excitar a produção de anticorpos (DE OLIVEIRA JA, et al., 2020).

Além do aumento no prognostico e na expectativa de vida dos portadores de HIV, nota-se que os aspectos psicológicos dos pacientes também são fortemente afetados pela descoberta e desenvolvimento da doença. Diante disso, a prática de atividade física diária pode ajudar na autoestima e autoconfiança dos infectados, pois eles possibilitam o afastamento dos efeitos negativos do estresse. Alguns hormônios que são liberados durante o exercício, como: o hormônio do crescimento $(\mathrm{GH})$, que proporciona a queima de gordura e funciona como agente anabólico; a endorfina, responsável por promover sensação de bem-estar e recompensa e está ligada ao sentimento de prazer e as catecolaminas que irão aumentar as taxas metabólicas, liberando glicose e ácidos graxos para a corrente sanguínea (IGNACIO DL e CONCEIÇÃO FL, 2018).

A prática de atividade física está sendo considerada uma das principais formas de promoção à saúde, além de prevenção de doenças cardiovasculares, câncer, osteoporose, obesidade, diabetes, entre outras. Diante disso, é possível destacar que as Unidades Básicas de Saúde (UBS), devem estar capacitadas e disponíveis para fazerem esse tipo de atendimento e dissipação dessa abordagem, a fim de engajar o maior número possível de pacientes. A criação do NASF, serviu para aumentar a resolutividade e a qualidade da Atenção Básica, proporcionando uma maior diversidade de profissionais de saúde que possam atender as mais diferenciadas necessidades, dentre eles estão os educadores físicos, nutricionistas, psicólogos, fisioterapeutas e médicos (KNOLL RK, et al., 2019).

A integração de um portador de HIV com a unidade de saúde, de nível primário, estabelece uma maior confiabilidade e formação de vínculos, favorecendo o tratamento e prosseguindo do doente dentro de um nível menos avançado, que consequentemente gera menos gastos e menor probabilidade de posteriores complicações. Os grupos direcionados a praticar exercícios na unidade de saúde ou caminhadas no próprio bairro, estão disponíveis para toda a população da localidade, sendo essa uma forma de promover a saúde de uma forma menos invasiva possível e evitando ou prevenindo o uso de medicação (PRIMEIRA MR, et al., 2020).

Além de todas essas contribuições geradas pelo exercício físico, adiciona-se a isso a melhoria na qualidade de vida das pessoas vivendo com HIV (PVHA), que engloba um bem-estar tanto físico quanto mental, além de relações interpessoais, sociais e individuais. Sendo assim essa prática possibilita a ampliação da inserção desses indivíduos na sociedade. Isso seria decorrente da mudança do estilo de vida, pois a partir disso terão uma maior socialização, ganho de autoestima e autoconfiança, se permitindo a conviver em diferentes contextos (MEDEIROS RCSC, et al., 2017).

Haverá também um aumento na expectativa de vida desses pacientes. Pois, a prática regular de atividade física possibilita uma redução nos problemas de saúde, como doenças cardiovasculares, diabetes, hipertensão e alterações relacionadas ao sistema imunológico trazendo consigo uma vida mais saudável. Ademais, o exercício aeróbico tem mostrado os seus benefícios em relação ao controle do estresse e como uma técnica de relaxamento capaz de diminuir a ansiedade e os sintomas depressivos (MEDEIROS RCSC, et al., 2017).

\section{CONSIDERAÇÕES FINAIS}

É notável os benefícios apresentados pela prática diária de atividade física, pois eles estão diretamente relacionados com a melhora e condicionamento do sistema imune do paciente portador de HIV. Ademais, conseguem promover uma dissipação de sentimentos que poderiam, posteriormente, acarretar transtornos mentais. Deve-se levar em consideração a importância do tratamento em conjunto, sendo tanto o tratamento medicamentoso quanto o não medicamentoso, para que haja um equilíbrio e uma maior oportunidade de melhora nas condições físicas e psíquicas do paciente. 


\section{REFERÊNCIAS}

1. BRITO CJ, et al. Impacto do treinamento resistido na força e hipertrofia muscular em HIV-soropositivos. Motriz: Revista de Educação Física, 2013;19: 313-324.

2. CARVALHO PP, et al. Fatores associados à adesão à Terapia Antirretroviral em adultos: revisão integrativa de literatura. Ciência \& Saúde Coletiva, 2019; 24: 2543-2555.

3. COUTINHO MFC, et al. Tratamento antirretroviral: adesão e a influência da depressão em usuários com HIV/AIDS atendidos na atenção primária. Saúde em debate, 2018; 42: 1-14.

4. CAMPOS KR, et al. Comparação de testes laboratoriais para o diagnóstico de infecção por vírus linfotrópicos de células T humanas do tipo 1 (HTLV-1) e tipo 2 (HTLV-2) em pacientes infectados por HIV-1. Revista do Instituto Adolfo Lutz, 2015; 74(1): 57-65.

5. DE MELO BO, et al. Epidemiologia e aspectos imunopatológicos do vírus da imunodeficiência humana (HIV): revisão de literatura. Revista Ceuma Perspectivas, 2018; 31(1): 86-100.

6. DA COSTA KM, et al. Alterações hematológicas, terapia antirretroviral e exercícios físicos: impacto no paciente soropositivo. Brazilian Journal of Clinical Analyses, 2018; 50(2): 60.

7. DE OLIVEIRA JA, et al. Influência do exercício físico sobre a contagem de células TCD4+ em pessoas vivendo com HIV/AIDS: uma revisão integrativa. Arquivos Brasileiros De Educação Física, 2020; 3(2): 50-55.

8. DA ROSA MC, et al. Patogênese do HIV - características do vírus e transmissão materno-infantil. Revista Brasileira de Análises Clínicas, 2016; 48(4): 301-6.

9. DIAS J, et al. Principais sintomas e alterações imunológicas decorrentes da infecção pelo vírus HIV: uma revisão bibliográfica. Revista Eletrônica Acervo Saúde, 2020; 40: e2715.

10. ERRANTE PR, et al. Antirretrovirais utilizados no controle da infecção pelo HIV. UNILUS Ensino e Pesquisa, $2018 ; 15$ (39): 114-130.

11. FREITAS JP, et al. Terapia com antirretrovirais: grau de adesão e a percepção dos indivíduos com HIV/Aids. Acta Paulista de Enfermagem, 2018; 31: 327-333.

12. GOUVÊA-E-SILVA LF, et al. Nível de atividade física e síndrome lipodistrófica em pacientes com HIV/aids. Revista Brasileira de Medicina do Esporte, 2016; 22: 147-152.

13. IGNACIO DL, CONCEIÇÃO FL. Hormônio do crescimento e exercício físico. Arquivos em Movimento, 2018; 14(1): $115-145$.

14. JUNIOR JO, et al. Adesão e aderência a um programa de exercício físico em pessoas vivendo com HIV/AIDS. Revista Brasileira de Atividade Física \& Saúde, 2017; 22 (6): 568-575.

15. KNOLL RK, et al. Práticas de uma equipe multiprofissional para pessoas vivendo com hiv/aids: um estudo de caso em um município da Foz do Rio Itajaí-açu, Santa Catarina-Brasil. Arquivos Catarinenses de Medicina, 2019; 48(4): 02-15.

16. LIMA ACMACC, et al. Construção e Validação de cartilha para prevenção da transmissão vertical do HIV. Acta Paulista de Enfermagem, 2017; 30(2): 181-189.

17. LIMA LRA, et al. Exercício Melhora o Risco Cardiovascular, Aptidão Física e Qualidade de Vida em Crianças e Adolescentes Hiv+: Estudo Piloto. International Journal of Cardiovascular Sciences, 2017; 30: 171-176.

18. LAZZAROTTO AR, BAZZO KO. Treinamento concorrente na imunidade e aptidão física de pacientes HIV/AIDS. Revista Brasileira de Medicina do Esporte, 2016; 22: 153-156.

19. LIMA ACMACC, et al. Transmissão vertical do HIV: reflexões para a promoção da saúde e cuidado de enfermagem. Avances en Enfermería, 2017; 35(2): 181-189.

20. MELO BP, et al. Respostas agudas do exercício físico em pessoas infectadas pelo HIV: uma revisão sistemática. Revista Brasileira de Medicina do Esporte, 2017; 23: 152-159.

21. MACIEL KL, et al. Estratégias de Assistência no Cuidado à Pessoa com Síndrome da Imunodeficiência Adquirida. Revista enfermagem atual, 2018; 86: 24.

22. MEDEIROS RCSC, et al. Qualidade de vida, fatores socioeconômicos e clínicos e prática de exercício físico em pessoas vivendo com HIV/aids. Revista de Saúde Pública, 2017; 51: 66.

23. NUNES AA, et al. Análise do perfil de pacientes com HIV/Aids hospitalizados após introdução da terapia antirretroviral (HAART). Revista de Ciência e saúde coletiva, 2015; 20(10): 3191-3198.

24. NUNES JÚNIOR SS, CIOSAK SI. Terapia antirretroviral para HIV/AIDS: o estado da arte. Revista de enfermagem UFPE on line, 2018; 12(4):1103-11.

25. PRIMEIRA MR, et al. Qualidade de vida, adesão e indicadores clínicos em pessoas vivendo com HIV. Acta Paulista de Enfermagem, 2020; 33: 1-8.

26. PINTO LFS, et al. Protocolo Brasileiro para Infecções Sexualmente Transmissíveis 2020: infecção pelo HIV em adolescentes e adultos. Epidemiologia e Serviços de Saúde. 2021, 30: e2020588.

27. PINTO CS, et al. Dinâmica temporal, subtipos e características epidemiológicas da infecção pelo hiv-1, Brasil Central. Rev. Saúde Pública Mato Grosso do Sul (Online), 2018; 1(1): 48-54.

28. PASSOS SMK, SOUZA LDM. An evaluation of quality of life and its determinants among people living with HIV/AIDS from Southern Brazil. Cadernos de saúde pública, 2015; 31: 800-814.

29. RODRIGUES DA, TOIGO AM. Efeitos de Diferentes Tipos de Exercício Físico em Portadores de HIV/AIDS com Lipodistrofia. Revista de Atenção à Saúde, 2015; 13: 73-78.

30. ROMANCINI JLH, et al. Níveis de atividade física e alterações metabólicas em pessoas vivendo com HIV/AIDS. Revista Brasileira de Medicina do Esporte, 2012; 18: 356-360.

31. SILVA RAR, et al. Conhecimento de estudantes adolescentes sobre transmissão, prevenção e comportamentos de risco em relação as DST/HIV/AIDS, 2016; 8(4): 5054-5061.

32. SOUZA HC, et al. Análise da adesão ao tratamento com antirretrovirais em pacientes com HIV/AIDS. Revista Brasileira de Enfermagem, 2019; 72: 1295-1303. 